\title{
Testing the feasibility of police provision of emergency contraception in Malawi
}

Malawi Human Rights Resource Centre

Malawi Police Service

Ministry of Health

Follow this and additional works at: https://knowledgecommons.popcouncil.org/departments_sbsr-rh

Part of the Demography, Population, and Ecology Commons, Domestic and Intimate Partner Violence Commons, Family, Life Course, and Society Commons, International Public Health Commons, Medicine and Health Commons, and the Women's Health Commons How does access to this work benefit you? Let us know!

\section{Recommended Citation}

Malawi Human Rights Resource Centre, Malawi Police Service, and Ministry of Health. 2012. "Testing the feasibility of police provision of emergency contraception in Malawi." Lilongwe: Malawi Human Rights Resource Centre. 
TESTING THE FEASIBILITY OF POLICE PROVISION OF

\section{EMERGENGY GONTRACEPTION} IN MAUAWI

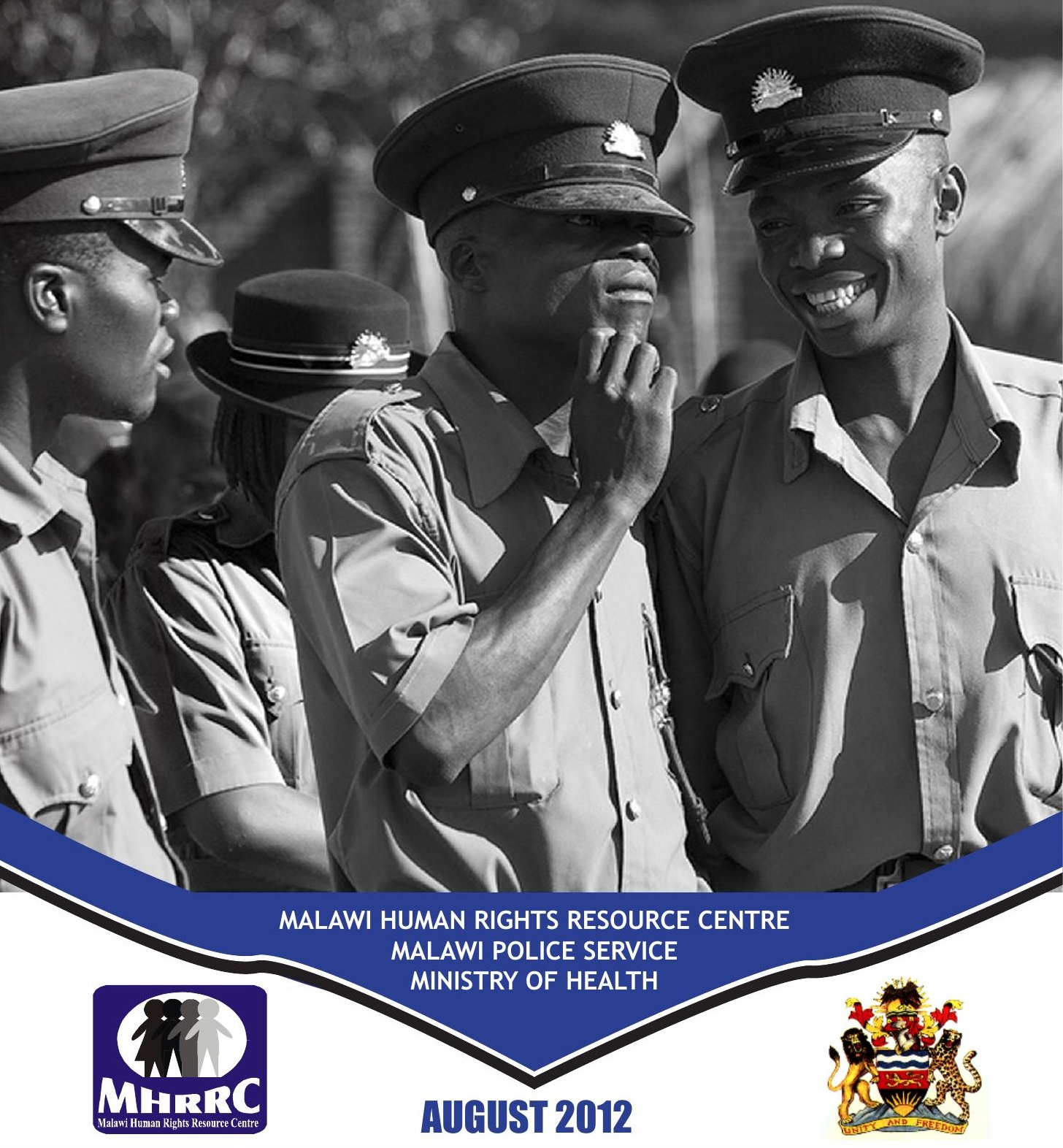




\section{Acknowledgements}

This project, Testing the Feasibility of Police Provision of Emergency Contraception in Malawi, was made possible with the help and cooperation of three institutions concerned for the plight of survivors of sexual assault: The Malawi Human Rights Resource Centre (MHRRC), the Malawi Police Service (MPS), the Ministry of Health and Population of the Government of Malawi (specifically, the Kamuzu Central Hospital-STI Department, the Reproductive Health Unit (RHU)), and the National Health Sciences Research Committee.

Special thanks go to the Inspector General of the Malawi Police Service for granting permission to have the project piloted at Lilongwe and Kanengo Stations, the Director of RHU for policy guidance, and the Lilongwe District Health Office for providing technical support as well as Emergency Contraception drugs for the project.

We also acknowledge the coordinating role played by management and staff of the MHRRC, mainly for providing office space, equipment, mobility, and material and logistical support during the course of the project.

We are grateful to informants for their cooperation, time and invaluable information provided. We are greatly indebted to the Zambian Copperbelt Model of Integrated Care for Survivors of Rape and Defilement (CMIC) program for synergising with the Malawi study team, and to the Population Council Lusaka and Nairobi offices for providing external learning opportunities to the study team at the Sub-Saharan Africa regional level.

We also acknowledge the efforts of all the persons who spared their time to read the draft report and provide input to improve its content and presentation.

This project was funded by the Swedish-Norwegian Regional HIV and AIDS Team for Africa, through the Population Council.

We hope that everyone who takes part in Sexual and Gender Based Violence (SGBV) will feel empowered to promote and protect the rights of women and girls, in particular, survivors of SGBV.
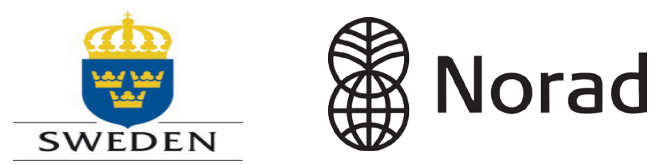

\section{(2) Population Council}




\section{Table of Contents}

Acknowledgements

Acronyms and Abbreviations

iii

Executive Summary

iv

Background

1

Project Goal

3

Project Sites

3

Project Activities

4

Ethical Considerations

7

Findings

7

Conclusions and Recommendations

Appendix 


\section{Acronyms and Abbreviations}

CMIC Copperbelt Model of Integrated Care

(for Survivors of Rape and Defilement)

DHO District Health Office

EC Emergency Contraception

$\mathrm{KCH} \quad$ Kamuzu Central Hospital

MHRRC Malawi Human Rights Resource Centre

$\mathrm{MOH} \quad$ Ministry of Health

PSC Project Steering Committee

RHU Reproductive Health Unit

SGBV Sexual and Gender-Based Violence

STI Sexually Transmitted Infection

UNFPA United Nations Population Fund

UNICEF United Nations Children's Fund

VSU Victim Support Unit 


\section{Executive Summary}

The overall goal of this project was to determine the feasibility of having trained police officers safely and effectively provide emergency contraception to survivors of sexual assault as a means of broadening access to comprehensive care. This effort was meant to capitalize on emerging findings in the region that the majority of survivors of sexual assault report to the police first, as well as to ensure immediate access to this critical element of post-rape care.

The project was implemented over a seventeen-month period (from April 2011 to August 2012) by the Malawi Human Rights Resource Centre (MHRRC) - a capacity-building non-government organization for Human Rights organisations in Malawi - in partnership with the Malawi Police Service (MPS), at Lilongwe and Kanengo Police Stations. The project was carried out in three phases: planning and materials development, training and project implementation, and data compilation and analysis.

Results from the project demonstrate that it is feasible to implement the police provision of EC model in Malawi. The police officers that participated in this project were able to effectively provide EC to eligible survivors, despite systemic barriers confronting police. The collaborative effort between police and health providers under the project initiated a process of referral strengthening between the Victim Support Units of Lilongwe and Kanengo Police Stations, and Kamuzu Central Hospital. There is, however, room for further strengthening of referrals between these two points to ensure optimal gains from this intervention. Although a proportion of survivors who accessed EC at police stations ended up using health care services as well, further efforts must be made to reduce barriers to seeking care after referral, and to increase the proportion of survivors doing so. Notably, the vast majority of survivors reporting to police stations during this project were children.

The project findings give rise to a number of recommendations, including the following:

1. SGBV needs to be better mainstreamed within police training and services.

2. Child-friendly services must be integrated into all levels of care for SGBV survivors.

3. Efforts should be made to enhance the referral process between police and health facilities.

4. A multi-sectoral training approach, involving the joint-training of police and health providers on critical documentation, is recommended to support this intervention. 


\section{Background}

Violence against women and children remains a major public health and human rights challenge in the world and Malawi in particular. The Government of Malawi recognizes that gender inequalities hinder the attainment of national socio-economic goals. Thus, it has put in place various supporting policies such as the National Gender Policy of 2000; the enactment of the Prevention of Domestic Violence Act, 2006; the Gender Equality Act, 2013; and the ratification of international conventions that promote the elimination of discrimination and violence against women and children. These efforts provide an enabling environment for the eradication of sexual and gender-based violence (SGBV).

Since 2010, the Malawi Human Rights Resource Centre (MHRRC), the Malawi Police Service, and the Malawi Ministry of Health $(\mathrm{MoH})$ have been partners under a Population Council program of technical assistance and research to strengthen the evidence base on SGBV programming in sub-Saharan Africa. Partners under this program are charged with developing, implementing and evaluating core elements of a comprehensive, multi-sectoral model for strengthening responses for survivors of SGBV, especially survivors of sexual violence. Lessons learned from across the continent through this program consistently indicate that police are the first, and often, only point of contact for survivors of sexual violence.

In South Africa, a record review demonstrated that $45 \%$ of all cases of domestic violence were initially reported to the police, and very few sought further health and legal services3. In Zambia, a similar review found that $91 \%$ of survivors reported first to the police, and only half as many presented for health care. Similarly, $92 \%$ of sexual violence cases seen at the Kamuzu Central Hospital in Malawi were referrals from the police (1). This reporting preference in the region has been attributed to a widespread perspective among survivors, police and health care providers of sexual violence as a criminal/legal issue alone, with the health implications often unknown (2). 
In Malawi, although survivors were successfully referred to Kamuzu Central Hospital from the police or reported there first, the hospital reported that a significant number of them presented outside the 72 hour window of opportunity for both emergency contraception (EC) and post-exposure prophylaxis (PEP) for HIV. This suggests that although survivors report to the police station and are referred to the health facilities within the timeframe for effective medical response, the survivors did not take advantage of the appropriate medical services.

Because the efficacy of EC is greatest when taken as soon as possible after unprotected sex (and no later than 120 hours), immediate access to EC is a critical element of quality post-rape care. Through the Population Council program, partners in Zambia broadened access to EC for sexual assault survivors through an intervention dubbed the 'Copperbelt Model of Integrated Care for Survivors of Rape and Defilement' (CMIC). This model demonstrated that, with training and support, police officers can safely and effectively provide EC to eligible women and girls (3). An evaluation of the study indicated that police provision of EC was successful in increasing survivors' access to the method, and community outreach activities undertaken during the implementation of the project increased awareness of these services.

The Malawi context was considered as being particularly appropriate for replicating this model given the cultural and administrative similarities between Zambia and Malawi, as well as the presence of a specialized Victim Support Unit in both countries that is mandated to handle all SGBV cases.

1. Selisho, Chali Chisala. 2010. "Malawi Case Study Report on the project: Towards a multisectoral approach on sexual and genderbased violence in Malawi." Lusaka: Population Council

2. ibid.

3. Keesbury, Jill, Mary Zama, and Sudha Shreeniwas. 2009. "The Copperbelt Model of Integrated Care for Survivors of Rape and Defilement: Testing the feasibility of police provision of emergency ccontraceptive pills." Lusaka: Population Council. 


\section{Project Goal}

The overall goal of this project was to determine the feasibility of having trained police officers safely and effectively provide emergency contraception to survivors of sexual assault as a means of broadening access to comprehensive care. Specifically, the project aimed to achieve the following results:

- Emergency contraception provided by VSU officers to eligible sexual assault survivors presenting at the Police station;

- Strengthened referrals between the VSU and the hospital;

- Increased utilization of health care services among the survivors who accessed EC at Police stations; and

- Feasibility of implementing the CMIC model in Malawi established.

\section{Project Sites}

The project was carried out in the Lilongwe Model Police Station, Kanengo Police Station, and Kamuzu Central Hospital $(\mathrm{KCH})$, all of which had strengthened SGBV response services to support the introduction of police EC provision. Lilongwe police station has a larger catchment area (4), serving both urban and rural communities, while Kanengo police station serves mainly peri-urban and rural catchment areas. The study also incorporated Kanengo's subpolice station of Lumbadzi, which serves a primarily rural area.

The referral point for the Police Stations was $\mathrm{KCH}$. $\mathrm{KCH}$ is the tertiary referral centre for the central region of Malawi, serving about 5 million persons. Services for sexual violence survivors are provided in the hospital's Sexually Transmitted Infections (STI) Clinic during the day and in the Gynaecology Department outside working hours. 


\section{Project Activities}

The project was carried out in three phases: planning and materials development, training and project implementation, and data compilation and analysis. These activities took place over a 17-month period, from April 2011 to August 2012.

\section{PLANNING AND MATERIALS DEVELOPMENT}

\section{Project Steering Committee Formation and regular meetings}

During the first quarter of the project, a stakeholder consultative meeting was held with the project partners and regulatory bodies, including the Ministry of Health Reproductive Health Unit (RHU), and the National Health Sciences Research Committee, to plan for the project and constitute the Project Steering Committee (PSC). The PSC also included members from the health sector, police, and Community Policing Department. The PSC met periodically during implementation to guide the project and develop strategies for sustainability.

\section{Procurement and Storage of EC drugs}

Despite inclusion in the Malawi National Guidelines for the Management of Sexual Assault and Rape, at the commencement of the project, EC drugs were not on the Essential Drug List for the Ministry of Health. They have since been included on the list, however.

The project started out (in the second and third quarters of the project period) by using Microgynon. Following this, and to ensure supply during the entire project, the Lilongwe District Health Office (DHO) procured a dedicated EC product (Pregnon) and donated 40 doses for use under the project once this drug was approved by the Ministry of Health.

The Research Nurse based at KCH's STI unit had the responsibility of overseeing the management of these supplies, ensuring that they were delivered and securely stored at the participating Police Stations, and providing technical support and quality assurance to ensure that the supplies were provided properly under Ministry of Health guidelines.

\section{Development of EC Logistics and Record Keeping Procedures}

EC supplies were stored in a lockable cabinet located in the Community Policing Department at the Police Stations. Only VSU officers trained to provide EC had access to the drug, and procedures were developed to ensure that trained officers were available on a 24-hour basis.

Two logs were also kept in the cabinet to ensure that EC was properly provided and recorded. These included a survivor log (a log book used to record all SGBV survivors seen by the VSU officers during the project period, regardless of whether they received EC or not) and a survivor 
screening checklist (a form used to determine survivors' eligibility for EC and also used as a job-aid to ensure that the VSU officers followed the required $\mathrm{MOH}$ protocol for providing EC).

\section{TRAINING AND PROJECT IMPLEMENTATION}

\section{Police training}

Materials to train the police to provide EC were adapted from the Zambia CMIC study. A multidisciplinary team comprising the MHRCC project coordinator, the STI Clinic Research Nurse, the District Family Planning Coordinator from the DHO, and the VSU project liaison officer conducted the police training.

Drawing on the experiences of the Zambia CMIC project, two types of trainings were conducted for different police audiences:

- Senior-level police officers (including shift supervisors) participated in a one-day sensitization on the project objectives, key issues in providing SGBV services, and the basics of EC provision as conducted by VSU officers. A total of 22 officers from across Lilongwe and Kanengo police stations participated in this training session.

- VSU officers participated in a 5-day training session that covered: basics on SGBV in Malawi, the law on various forms of SGBV, the needs of an SGBV survivor, services available at the hospital (including a site visit to $\mathrm{KCH}$ ), EC as a method of preventing pregnancy after sexual assault, doses and brands available in Malawi, efficacy and side effects, provision protocol, and referral procedures. A total of 16 VSU officers from across Lilongwe and Kanengo police stations participated in this training session.

\section{Training for Health Care Workers}

A limited number of providers are trained in SGBV care in the region. To address this, MHRCC collaborated with South Africa's Medical Research Council to conduct a five-day training session for health care providers at $\mathrm{KCH}$. A total of 16 doctors, nurses and clinical officers participated in this training which supported the project by strengthening health care services in the referral facility.

\section{Implementation and supportive supervision}

Once VSU officers and health care staff were trained, they began providing SGBV services with support from the project team. Police provision of EC received periodic support from the project team in the first month of implementation to ensure that the drug was being properly provided. Throughout the project, the MHRCC project coordinator and the STI Research Nurse conducted periodic visits to participating police stations to review the survivor logs and survivor screening checklists, address any questions or concerns that arose, and provide technical support to the officers. They were supported during these visits by the VSU Liaison Officer. 
At $\mathrm{KCH}$, the STI Clinic nurse provided day-to-day support to efforts to improve after-hour services and to ensure the provision of quality SGBV services by the STI Clinic.

\section{Community Awareness Activities}

In order to increase awareness of SGBV and the services available to survivors, including EC, the project also worked in the communities surrounding the police stations. Based on the Zambia CMIC experience, this was expected to promote utilization of both police and health care services, and increase reporting within 72 hours of sexual assault.

The Malawi Police VSU has a presence in the project communities through community structures referred to as Community Victim Support Committees. These committees are the "eyes and ears" of the police at community level and are very active, conducting sensitization and referring survivors to the police stations. The police made use of these structures to disseminate information on the availability of EC at the police stations and the need to report SV cases as soon as possible in order to take advantage of such available services. MHRRC also made use of existing Community Action Groups, Radio Listening Clubs and the Men's Network to sensitize the communities.

\section{DATA COMPILATION AND ANALYSIS}

Program evaluation data were collected from the following sources: survivor log books, survivor screening checklists, STI Clinic and Gynaecology Department records, and key informant interviews.

On a monthly basis, the MHRRC program coordinator transcribed data (service statistics) from the survivor log books and collected the survivor screening checklists. During the transcription process, each survivor was assigned a unique code to ensure anonymity while allowing for identification in health facility records. No names were transcribed.

To measure referrals and the improvement of care at the health facility, the STI Clinic Research Nurse reviewed records from the STI Clinic and the Gynaecology Department on a monthly basis and transcribed the data generated from these record reviews. Data entry and analysis were then carried out at the MHRRC office.

At the end of the project, a total of 5 key informant interviews were conducted with representatives of the STI Clinic at $\mathrm{KCH}$, the Community Policing Department at the National Police Headquarters, the Kanengo and Lilongwe Victim Support Units, and the MHRRC management. These interviews assessed perceptions of the impact of the police provision of EC model and its ability to be sustained within the public sector. All interviews were audiotaped, transcribed and manually coded. 


\section{Ethical Bonsiderations}

No personal identifiers were collected from SV survivor's records. Rather, as earlier noted, a unique code that enabled MHRRC to link survivor records from police stations to the health facility was generated for each survivor. All data were stored securely at the MHRRC office in a locked cabinet, with restricted access to the database. At the police station, all project records were kept in a lockable cabinet accessible by trained VSU officers and the MHRRC program coordinator only. Prior to participating in the key informant interviews, all participants were provided with an informed consent form and given the opportunity to decline. The National Health Sciences Research Committee in Malawi provided ethical and research clearance for the project.

\section{Findings}

A low number of police officers have received SGBV-related training

There was a low number of available Police Officers in the participating stations (Table 1), considering the scope of area under their jurisdiction. Even fewer were specifically trained as VSU officers. Of the 33 police officers stationed within the police sites, about half were trained in EC provision, with representation by both female and male officers. Data from key informant interviews suggest a tendency to redeploy officers trained in EC provision to perform other, 'core' police duties, such as public order management, thus possibly narrowing access to services for survivors. The frequent transfer of police officers (including VSU officers trained in EC provision and the management of sexual assault) was also perceived as contributing to this dynamic.

\begin{tabular}{|c|c|c|c|c|}
\hline Personnel Capacity & \multicolumn{2}{|c|}{$\begin{array}{l}\text { Lilongwe } \\
\text { male female }\end{array}$} & \multicolumn{2}{|c|}{$\begin{array}{l}\text { Kanengo } \\
\text { male female }\end{array}$} \\
\hline $\begin{array}{l}\text { \# of officers trained by } \\
\text { Medical Research Council }\end{array}$ & 7 & 11 & 11 & 4 \\
\hline \# of trained VSU officers & 2 & 2 & 4 & 4 \\
\hline $\begin{array}{l}\text { \# of officers trained in } \\
\text { EC provision }\end{array}$ & 5 & 5 & 3 & 3 \\
\hline Totals & 14 & 18 & 18 & 11 \\
\hline
\end{tabular}


EC was provided effectively by police

Despite the limitations in the number of police offers that had received SGBV training, over the life of the project (from April 2011 to September 2012), a total of 37 doses were administered to qualifying survivors of sexual assault by police at the two police stations without the emergence of adverse events. Ten doses of Microgynon were administered in the second and third quarters of the project before Pregnon was approved by the Ministry of Health. Once Pregnon was approved, 27 doses of this drug were administered by police as at September 2012. In accordance with the project protocol, EC was only given to women and girls of reproductive age, and no younger than 11 years of age.

Similar to the Zambia CMIC study, these findings point to the feasibility of police provision of $\mathrm{EC}$ to survivors of sexual violence in a safe and effective manner.

\section{The majority of survivors reporting to the hospital for care were minors}

From January to June, 2012, a total of 54 survivors reported to KCH for SGBV services (Table 2). Of these, $80 \%$ were children and young people aged 18 years and below. This finding is consistent with that of other studies in the region that have indicated sexual violence involving children and youth is more likely to be reported. (5) This also suggests significant underreporting on the part of women who have experienced sexual violence.

Due to documentation inconsistencies, it is unclear whether all 54 survivors presented at $\mathrm{KCH}$ as a result of referrals from the police, or whether some survivors sought services at $\mathrm{KCH}$ independently.

Table 2: Age range of survivors presenting at $\mathrm{KCH}$ (January-June 2012)

\begin{tabular}{l|l|l|l}
\hline Age range & $0-18$ & & 19 and above \\
\hline Frequency & 43 & & 11 \\
\hline Total & & 54 & \\
\hline
\end{tabular}

\section{Police provided consistent referrals}

Between January and June, 2012, a total of eighteen (18) survivors received EC at the police stations, while forty-nine (49) cases were referred from the two police stations (Kanengo and Lilongwe) to KCH's STI Department for comprehensive care (Table 3). The discrepancy in the number of clients receiving EC versus the number being referred has to do with the fact that survivors reporting to the police tended to be children. From January to June, 2012, the majority of survivors reporting to the participating police stations were also below the age of 11 , meaning that they were ineligible to receive EC under the project

5. Keesbury, Jill, Mary Zama, and Sudha Shreeniwas. 2009. "The Copperbelt Model of Integrated Care for Survivors of Rape and Defilement: Testing the feasibility of police provision of emergency contraceptive pills." Lusaka: Population Council; Keesbury, J \& Askew, I. (2011). Comprehensive responses to sexual violence in East and Southern Africa: Lessons learned from implementation. Lusaka: Population Council. www.popcouncil.org/pdfs/2010RH_CompRespGBV.pdf.; Ajema C, Mukoma W, Mugyenyi C, Meme M, Kotut R, and Mulwa R (2012). Improving the collection, documentation and utilisation of medico-legal evidence in Kenya; LVCT Kenya. 
Table 3: Client flow from police to KCH (January to June, 2012)

\# of cases that received EC from police \# of cases referred by police to $\mathrm{KCH}$ \# of cases received at $\mathrm{KCH}$ through police referral

\begin{tabular}{|c|c|c|c|c|c|c|}
\hline Month & & & & & & \\
\hline $\begin{array}{l}\text { January } \\
\text { February } \\
\text { March } \\
\text { April } \\
\text { May } \\
\text { June }\end{array}$ & $\begin{array}{l}1 \\
1 \\
0 \\
1 \\
3 \\
0\end{array}$ & $\begin{array}{l}3 \\
4 \\
3 \\
0 \\
2 \\
0\end{array}$ & $\begin{array}{l}6 \\
7 \\
3 \\
3 \\
4 \\
2\end{array}$ & $\begin{array}{l}5 \\
3 \\
3 \\
5 \\
6 \\
2\end{array}$ & $\begin{array}{l}2 \\
0 \\
2 \\
0 \\
0 \\
0\end{array}$ & $\begin{array}{l}2 \\
0 \\
0 \\
4 \\
2 \\
1\end{array}$ \\
\hline Total & & & & & & \\
\hline
\end{tabular}

The rate of referral uptake by survivors was low

Although the police were able to provide EC properly and were consistent in referring survivors for further care at $\mathrm{KCH}$, the proportion of survivors that took advantage of these referrals remained low. Of the 49 cases referred to KCH by the police between January and June 2012, only $27 \%(n=13)$ subsequently sought comprehensive care at $\mathrm{KCH}$ (Table 3$)$.

Several key informants also noted that some survivors present at the hospital too late, thus limiting the kind of services they can receive, such as post-exposure prophylaxis for HIV and forensic tests. Although services are provided to survivors for free at $\mathrm{KCH}$, transportation (between the police stations and hospital) was observed as a major barrier to the uptake of the referrals.

\section{Strengthening of documentation is required}

Consistent documentation or record-keeping is a key requirement to support this intervention. A review of project records suggests that, while documentation of survivor in-take and referrals was occurring, this was not always consistent across the police sites. Key informant interview data substantiate this finding, with respondents indicating that police referral forms did not always indicate whether survivors had received EC or not. In some cases, dates of sexual assault incidents were not recorded. Yet, complete and consistent documentation by providers is critical for monitoring progress and for understanding how best to support survivors. 
Key informants observed that over the project period, for the first time ever, there were several reports of intimate partner violence (marital rape in particular). Key informants linked this new phenomenon to the community sensitization exercises carried out by police as part of the intervention. It was noted that communities seemed to be more aware of SGBV and felt more comfortable reporting to the police.

There was evidence of preparedness by key sectors and partners to replicate and/or scale up the intervention

All the VSU officers interviewed saw both the need and opportunities for replicating or scaling up this project beyond the original pilot sites. Partners that have been part of the Project Steering Committee have also expressed in supporting the project. Avenues to promote replication and scale-up were identified, including: engaging with the top management within the Ministry of Health, and with UNICEF, both of which have shown keen interest in the project; and engaging with UNFPA, which recently organized EC training for health care workers in Lilongwe and Mchinji. 


\section{Ponclusions and Recommendations}

This project aimed to determine the feasibility of having trained police officers safely and effectively provide emergency contraception to survivors of sexual assault as a means of broadening access to comprehensive care.

Project results have helped to establish the feasibility of implementing this police provision of EC model in Malawi. The police that participated in this project were able to effectively provide EC to eligible survivors. The collaborative effort between police and health providers under this project has initiated a process of referral strengthening between the Victim Support Units of Lilongwe and Kanengo police stations, and Kamuzu Central Hospital. There is, however, room for further strengthening of referrals between these two points to ensure optimal gains from this intervention. Although a proportion of survivors who accessed EC at police stations ended up using health care services as well, further efforts must be made to reduce barriers to seeking care after referral, and to increase the proportion survivors doing so.

The primary recommendations based on the project results are as follows:

1. SGBV needs to be better mainstreamed within police training and services. Frequent police transfers, the perception among police that attending to SGBV issues is the preserve of VSU officers alone, and the perception of SGBV work as not being at the 'core' of police duties emerged as issues facing the police. Mainstreaming SGBV in both police training and services can help to mitigate these issues. Police provision of EC and the management of sexual assault would form part of the mainstreaming process.

2. Child-friendly services must be integrated into all levels of care for SGBV survivors. The majority of survivors seeking services at the police stations and hospital were children. This reality must inform the organization of services for SGBV survivors. Childfocused services must be integrated into all related sectors, include police, health (including psychosocial services), and social welfare. Such services also require the recruitment and/or training of staff to attend to children in particular. 
3. Efforts should be made to enhance the referral process. The number of referred survivors that sought comprehensive care at the hospital was far fewer than the number actually referred to the hospital by the police. Potential barriers to referral uptake, such as inadequate information, transportation, and costs, should be addressed. As community sensitization appeared to reap positive results, this medium may be used to educate the community on the health implications of SGBV.

4. A multi-sectoral training approach is recommended to support this intervention. Proper documentation of client flow by police and health providers is a key part of the intervention process. To facilitate this, police and health providers alike must understand the role of documentation in the proper care of survivors. Joint trainings between police and health providers (focusing on documentation) have been shown to be particularly effective, (6) and should be employed to facilitate intervention monitoring. Special, standard registers for SGBV (as opposed to improvised versions) can also help improve documentation, while institutionalizing care of survivors in police stations.

6. Ajema C, Mukoma W, Mugyenyi C, Meme M, Kotut R, and Mulwa R (2012). Improving the collection, documentation and utilisation of medico-legal evidence in Kenya; LVCT Kenya. 


\section{Appendix}

Client Screening Checklist for Police Provision of EC

- Complete all questions to determine a client's eligibility for EC

- Place all completed forms in the EC storage container. They will be collected by the district family planning coordinator

Facility

Date form completed

VSU Officer Case O.B. Number

Client's Age 
1. Was the client raped/ defiled?

Yes No

(go to 2 ) Client not eligible

2. Did the assault involve penetrative sex? Yes

(go to 3) Client not eligible

3. Did the assault occur within the past 120 hours

Yes

(go to 4)

No

Client not eligible

4. Has the client had her first menstrual period?

Yes

(go to 5)

No

Client not eligible

5. Does the client want to

Yes

(Go to 6)

No

prevent pregnancy?

Client not eligible

If client answered "YES" to questions 1-5, she is eligible for EC. Proceed.

If client answered 'NO" to ANY of the questions above, she is not eligible. STOP!

6. Is the client currently pregnant?

$\begin{array}{ll}\text { Yes } & \text { No } \\ \text { (go to 7) } & \text { Client not eligible }\end{array}$

If the client's pregnancy status is unclear, EC may still be given, but with the explanation that it will not work if she is already pregnant and will not harm the foetus.

7. Was the client's last menstrual period less than 4 weeks ago

Yes

(go to 8 )
NO

Do not dispense

If client answered "yes" to questions 7 and 8, EC may be provided

*adopted from: The CMIC Model, Zambia 

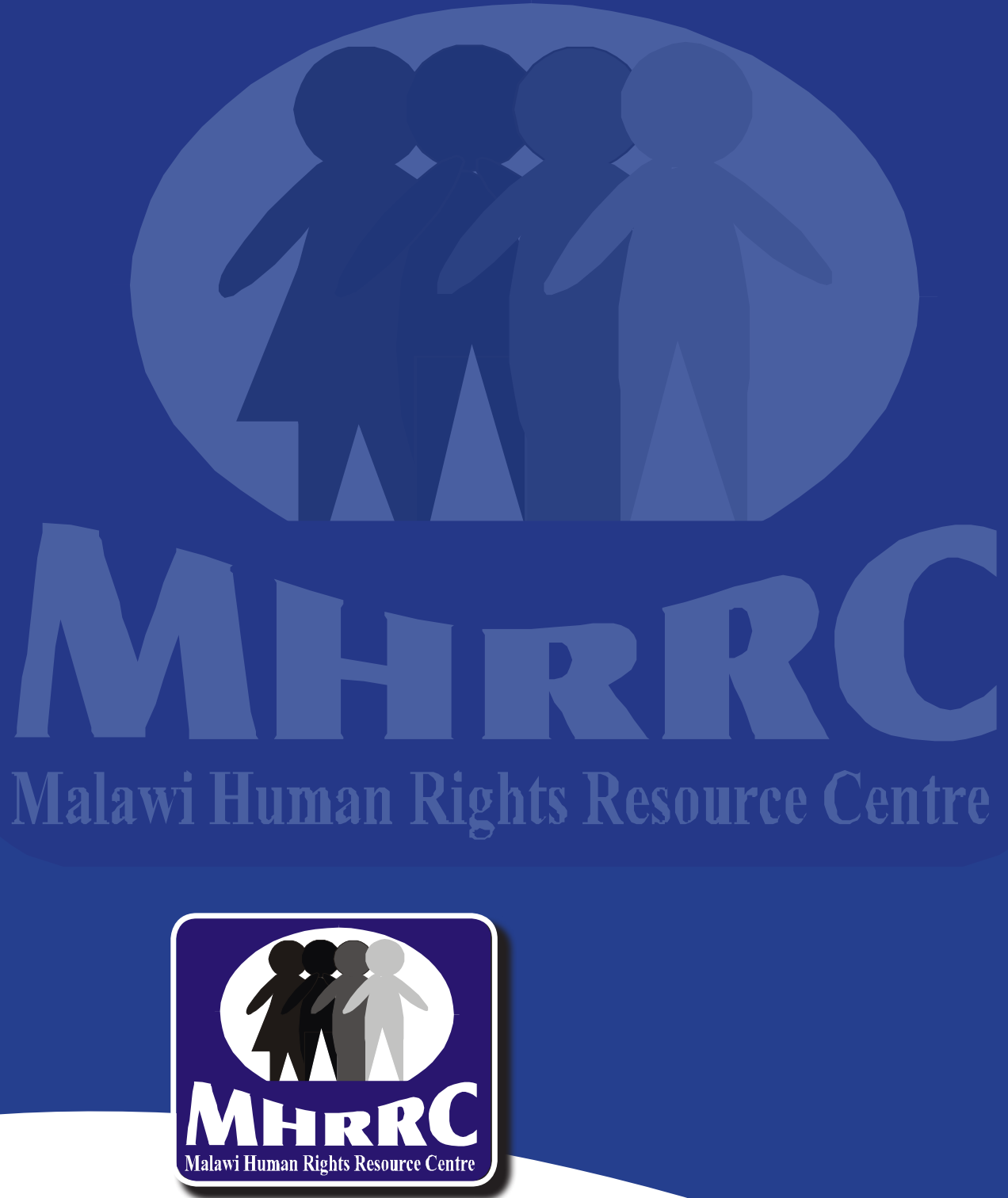

Malawi Human Rights Resource Centre Area 9 (Plot No. 9/190), P.O. Box 891, Lilongwe. Tel.: (+265) 1752629 Fax: (+265) 1751390 E-mail: info@humanrights.mw Website: www.humanrights.mw 